hautnah $2013 \cdot 12: 23-23$

DOI 10.1007/s12326-013-0045-5

C) Springer-Verlag Wien 2013
E. Tschachler

Forschungsabteilung für Biologie und Pathobiologie der Haut, Universitätsklinik

für Dermatologie, Medizinische Universität Wien, Wien

\title{
Das Mikrobiom des Menschen
}

\section{Eine Grenzfläche zwischen Gesundheit und Krankheit}

\begin{abstract}
Was haben die seborrhoische Dermatitis, die Akne vulgaris und die Rosacea gemeinsam? Bei allen drei Erkrankungen spielen Mikroorganismen, die bereits unter normalen Bedingungen die gesunde Haut besiedeln, eine pathogenetische Rolle. Wie es dazu kommt, dass Keime der Mikroflora plötzlich krankheitsrelevant werden können und was die Bedeutung ebendieser Keime für die Gesundheit des Hautorgans ist, wurde in den letzten Jahren zunehmend zu einem zentralen Forschungsgebiet.
\end{abstract}

\section{Genaue qualitative und quantitative Erfassung}

Die atemberaubende Entwicklung molekularer Analysemethoden in den letzten Jahren hat es ermöglicht, die Gesamtheit der Keime, i. e. das Mikrobiom, ein gegebenes Habitat in unserem Körper, qualitativ und quantitativ zu erfassen und damit ein erste Erkenntnisse über den Beitrag residenter Mikroorganismen zu Krankheitsprozessen zu erlangen. Es ist mittlerweile bekannt, dass in unserem Organismus mehr Bakterienzellen als köpereigene Zellen leben, dass die bakterielle Besiedelung gesunder Menschen große Unterschiede aufweist und dass sie das Leben ihres Wirtes maßgeblich beeinflussen kann. So gibt es inzwischen starke Hinweise darauf, dass residente Darmbakterien sowohl an metabolischen Dysregulationen, die zur Adipositas führen, als auch an entzündlichen Darmerkrankungen sowie an der funktionellen
Ausbildung des Immunsystems direkt und indirekt beteiligt sind.

\section{Unterschiedliche \\ Zusammensetzung}

Durch die Pionierarbeit von Julia Segre und ihrer Arbeitsgruppe an den National Institutes of Health in den USA steht seit Kurzem ein Katalog der residenten Bakterien der verschiedenen Hautregionen zur Verfügung, und es hat sich gezeigt, dass zwischen verschiedenen Menschen große Unterschiede in der Zusammensetzung des Mikrobioms der Haut bestehen.

\section{Klinische Relevanz}

Mittlerweile liegen auch erste Untersuchungen mit unmittelbarer klinischer Relevanz vor. So nimmt etwa die Diversität des Mikrobioms der Haut bei Patienten mit atopischer Dermatitis in der Krankheitsphase zugunsten eines Überwiegens von Staphylococcus aureus stark ab. Diese Veränderung, die auch ein Rezidiv ankündigt, kann durch intermittierende antibiotische oder antiinflammatorische Therapie verzögert werden.

\section{Schutz vor Besiedelung mit pathogenen Keimen}

Die Fähigkeit residenter Bakterien, die Kolonisierung mit pathogenen Bakterien $\mathrm{zu}$ verhindern, ist schon seit Langem bekannt - die Mechanismen dieser positiven Wirkung lagen jedoch noch im Dunkeln. Ge- rade in Bezug auf S. aureus, der Keim, der am häufigsten Hautinfektionen verursacht, haben sich in den letzten Jahren faszinierende Erkenntnisse ergeben. Bestimmte Stämme des Hautkommensalen S. epidermidis sind in der Lage, einer Besiedelung durch den pathogenen S. aureus entgegenzuwirken. Diese Fähigkeit beruht auf der Sekretion eines proteolytischen Enzyms durch S. epidermidis, welches die Biofilmbildung von $S$. aureus und damit die Kolonisierung mit diesem Keim verhindert. Durch Applikation des rekombinanten Enzyms in die Naseneingänge von S.-aureusTrägern konnte der pathogene Keim innerhalb von Tagen eliminiert werden. Dasselbe Ergebnis konnte durch die Inokulation des S.-epidermidis-Stammes, der dieses Enzym produziert, erreicht werden. In Anbetracht der steigenden Gefahr von Infektionen durch Methicillin-resistente S.-aureusStämme stellt diese Entdeckung eine bedeutende Erweiterung unseres antimikrobiellen Armentariums dar und leitet vermutlich eine neue Ära der „biologischen Kriegsführung " in der Medizin ein.

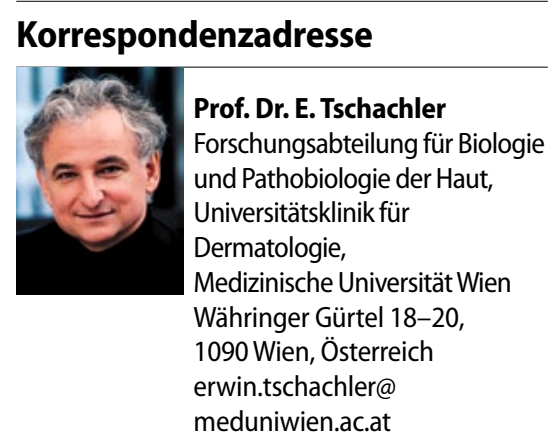

